\title{
Retroviral activation of the mir-106a microRNA cistron in $\mathbf{T}$ lymphoma
}

\author{
Amy M Lum¹, Bruce B Wang1, Lauri Li ${ }^{1}$, Namitha Channa ${ }^{1}$, Gabor Bartha ${ }^{2}$ \\ and Matthias Wabl*3
}

\author{
Address: ${ }^{1}$ Picobella, L.L.C., 863 Mitten Road, Suite 101, Burlingame, CA, 94010, USA, ${ }^{2}$ Synergenics, L.L.C., 863 Mitten Road, Suite 101, \\ Burlingame, CA, 94010, USA and 32Department of Microbiology and Immunology, University of California, San Francisco, CA, 94143-0414, USA \\ Email: Amy M Lum - amy@picobella.com; Bruce B Wang - bruce@picobella.com; Lauri Li - lauri@picobella.com; \\ Namitha Channa - namitha@picobella.com; Gabor Bartha - gabor@synergenics.net; Matthias Wabl* - mutator@ucsf.edu \\ * Corresponding author
}

Published: 25 January 2007

Retrovirology 2007, 4:5 doi:10.1186/1742-4690-4-5

This article is available from: http://www.retrovirology.com/content/4/1/5

(c) 2007 Lum et al; licensee BioMed Central Ltd.

This is an Open Access article distributed under the terms of the Creative Commons Attribution License (http://creativecommons.org/licenses/by/2.0), which permits unrestricted use, distribution, and reproduction in any medium, provided the original work is properly cited.
Received: 12 December 2006

Accepted: 25 January 2007

\begin{abstract}
Retroviral insertion into a host genome is a powerful tool not only for the discovery of cancer genes, but also for the discovery of potential oncogenic noncoding RNAs. In a large-scale mouse T lymphocyte tumor screen we found a high density of integrations upstream of the mir-106a microRNA cistron. In tumors containing an integration, the primary transcript encoding the mir106a cistron was overexpressed five to 20-fold compared with that of control tumors; concomitantly, the mature mir-106a and mir-363 microRNAs were highly overexpressed as well. These findings suggest the mir- I06a cistron plays an important role in $\mathrm{T}$ cell tumorigenesis.
\end{abstract}

\section{Findings}

Retroviral insertions into the genome of a host can induce tumor formation by altering gene expression or function. Integration of a retrovirus near a gene can induce overexpression of the gene through the viral promoter or enhancer, while insertion of a retrovirus into a gene can cause both activation and inactivation. If the affected genes are proto-oncogenes or tumor suppressor genes, the insertion events may lead to tumor formation [1]. Consequently, retroviral mutagenesis has been used to search entire genomes for genes involved in cancer development [2-4], including oncogenic microRNAs (miRNAs) [5]. MiRNAs are short ( $22 \mathrm{bp}$ ) noncoding RNAs that are implicated in gene regulation and cancer [6-10]. In a large-scale retroviral insertion mutagenesis screen, we used the murine leukemia virus (MLV) strain SL3-3, which causes $\mathrm{T}$ lymphomas [11], and identified several miRNAs that are potentially involved in tumorigenesis.
We previously demonstrated that a group of these retroviral insertions induces overexpression of the oncogenic mmu-mir-17 miRNA cistron in mouse tumors [5]. Here we build on our validation of the retrovirus insertional mutagenesis method to identify oncogenic miRNA and present another potentially oncogenic miRNA cistron, mmu-mir-106a. In this screen, male BALB/c mice were treated with ethyl-nitroso-urea (ENU) and bred to normal female mice. ENU treatment was conducted to increase the recovery of tumor suppressors in the F1 progeny through mutagenesis of the paternal allele. Newborn offspring mice were then injected with MLV strain SL3-3. After becoming moribund due to tumor development, mice were euthanized and thymus and spleen tissues were collected and stored at $-80^{\circ} \mathrm{C}$. Locations of the SL3-3 provirus integration sites were identified as previously described using a splinkerette based PCR method [3] that amplifies genomic DNA flanking the 5' LTR of the virus. 
We identified 6234 integration sites in 2199 tumors; of these tumors, 76 sites were located on chromosome $\mathrm{X}$ upstream of a miRNA cluster containing mmu-mir-106a, mmu-mir-20b, mmu-mir-19b-2, mmu-mir-92-2, and mmu-mir-363. The locations of the integrations ranged from $1.5 \mathrm{~kb}$ to $22 \mathrm{~kb}$ upstream of the miRNA cluster (Fig. 1 ), with proviral inserts in both sense and anti-sense orientations with respect to the primary RNA transcript encoding the miRNA cistron. The Mouse Retroviral Tagged Cancer Gene Database [12], which compiles retroviral insertions into the genomic DNA from various non$\mathrm{T}$ cell derived mouse tumors, also lists 10 integrations located upstream of the mmu-mir-106a cluster. Furthermore, Hwang et al. found that EST AI464896, which maps to the same location as mmu-mir-363, was overexpressed in tumors with proviral MLV integrations into this region [13]. The radiation leukemia virus (RadLV) also frequently integrates at this locus and a group of five differentially spliced noncoding RNAs known as Kis2 (GenBank Accession numbers AY940614-AY940618) are overexpressed in these tumors [14]. Because the Kis2 transcripts lie directly upstream of the mir-106a miRNA cluster (mmu-mir-106a overlaps these transcripts by four bases), they likely are part of the primary transcripts containing the miRNA cluster.

To determine whether the retroviral integrations in this region affected the expression of the mir-106a cistron, we used quantitative PCR (qPCR) to measure expression levels of the primary transcript (Kis2) and the mature miRNAs (mmu-mir-106a and mmu-mir-363) in tumors containing mir-106a cistron integrations as well as in control tumors lacking such integrations. To measure primary transcript (Kis2) expression levels, a probe and primer set was designed to AY940616, which is a common exon to three of the alternatively spliced forms of Kis2. The probe and primers for AY940616 were as follows: 5'-TGTGTCCCTGAAGTTTATTGGTGT-3', 5'-GGGTCACGAGCTCCCTCC-3', and 5'-[6-FAM]CCCCCATCAACACAAACATTCCATCA-[3BHQ1]-3'. MiRNAs and low molecular weight RNAs were isolated from frozen mouse tumor tissue using the Purelink miRNA Isolation Kit (Invitrogen). Large fraction RNAs were then purified by eluting the high molecular weight RNA bound to the first column (used for the miRNA purification). cDNA was generated from total RNA by reverse transcription with random hexamers using the SuperScript FirstStrand Synthesis System for RT-PCR (Invitrogen). qPCR runs were conducted on the MX3000P (Stratagene). All qPCR reactions were run in triplicate. As controls, tumors not containing integrations near the mmu-mir-106a-363 cluster were also assayed. Beta-actin was used as the endogenous reference gene (Mouse ACTB 20× VIC-MGB probe set, Applied Biosystems) and control tumor 1 was used as the calibrator sample in the calculation of $2^{-\Delta \Delta \mathrm{Ct}}$ values (relative expression). All relative expression values were normalized such that the average of the tumor controls was set to 1 .

Representative tumors with integration sites spanning the upstream region of mir-106a were measured for expression of the miRNA primary transcript (Fig. 1 and Table 1). In 16 of the 21 tumors assayed, expression of AY940616 was elevated five to 20 fold as compared to the average expression of tumors with no integrations at this locus (Fig. 2A). This confirms the previous report that proviral integrations in this region can increase expression of the Kis2 locus [14].

The mature species of mmu-mir-106a and mmu-mir-363 were then measured by RT-qPCR using a stem-loop RT primer specific for each miRNA [15]. Accordingly, $50 \mathrm{ng}$ of each tumor miRNA preparation was reverse transcribed with the SuperScript First-Strand Synthesis System for RTPCR using the following stem loop RT primers $(50 \mathrm{nM}$ final concentration) 5'-GTCGTATCCAGTGCAGGGTCCGAGGTATTCGCACTGGATACGACTACCTG-3'(mmumir-106a) and 5'-GTCGTATCCAGTGCAGGGTCCGAGGTATTCGCACTGGATACGACTTACAG-3' (mmu-mir-363). The reverse transcription reactions were diluted 1:200 and $5 \mu \mathrm{l}$ of these dilutions were used in the $25 \mu \mathrm{l}$ qPCR reactions. The annealing step was $50^{\circ} \mathrm{C}$ for $60 \mathrm{~s}$. The qPCR probes and primers were as follows: mmu-mir-106a: $5^{\prime}-$ CGGCAAAGTGCTAACAGT-3', 5'-GTGCAGGGTCCGAGGT-3', 5'- [6-FAM]- CACTGGATACGACTACCTGC[BHQ1]-3'; and mmu-mir-363: 5'-TGCGGATTGCACGGTATC-3', 5'-GTGCAGGGTCCGAGGT-3', 5'- [6-FAM]CACTGGATACGACTTACAGATG- [BHQ1]-3'. Synthetic RNA oligos (IDT) were used to generate a calibration curve for each miRNA: 5'-CAAAGUGCUAACAGUGCAGGUA-3' (mmu-mir-106a) and 5'-AUUGCACGGUAUCCAUCUGUAA-3'(mmu-mir-363). Amplification efficiencies of the calibration curves for mmu-mir-106a and mmu-mir-363 were respectively $67 \%$ and $69 \%$. Concentrations of the mature species were calculated using the calibration curves and then normalized by the average of the control tumors, to calculate relative expression levels.

Fifteen tumors with integrations in this region were assayed by qPCR for the mature species of mmu-mir-106a and mmu-mir-363. Approximately $70 \%$ of these tumors had increased expression levels of mmu-mir-106a by two to six fold, and of mmu-mir-363 by four to 12 fold over the average expression of tumors with no integrations in this region (Fig. 2B). The mature miRNA expression difference between tumors with integrations in this region and the tumor controls was statistically significant $[\mathrm{p}<$ 0.00001 (mmu-mir-106a) and $\mathrm{p}<0.0001$ (mmu-mir363)] by a two sample unequal variance Student's test. From these data we conclude that retroviral integrations 


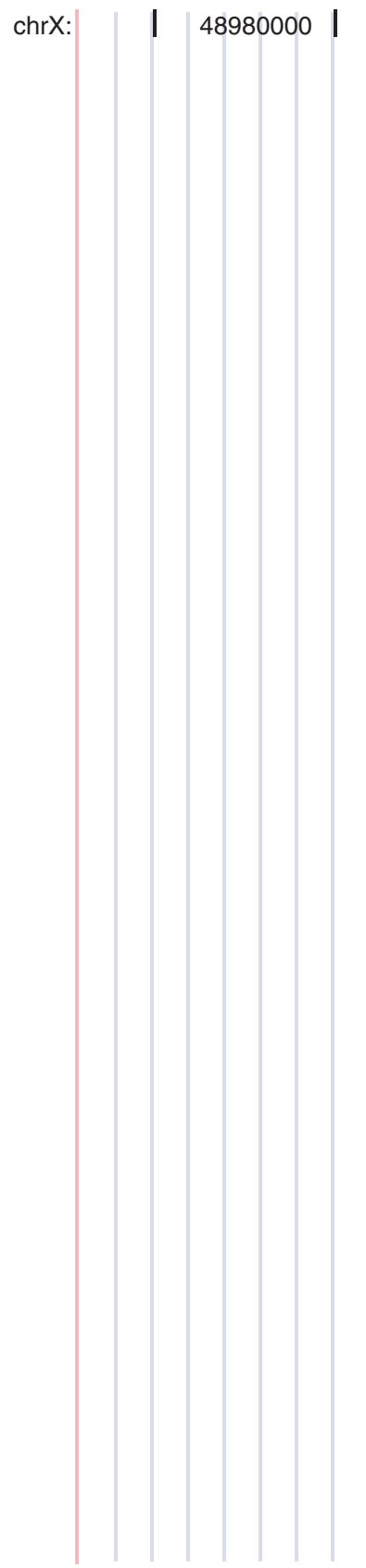

48985000 | 48990000 |

$1505 \mathrm{~S}-213-3-1$

(1) 1759S-158-18-4

(2) 1890S-76-1-1 ||

(3) 718S-68-23-3 ||

(4)

1786S-166-32-3

614S-155-3-5 ||

614T-155-3-4

1276S-152-28-1 ||

(5)

818S-36-8-3

1818S-176-3-3 ||

1792T-171-13-2

553S-122-16-2

(7) 1758S-158-17-2

1554S-139-23-1 ||

2020S-213-11-1 ||

(8) 1189S-187-12-1

(9) 3390T-128-37-1

809S-6-53-1

2141S-154-28-2

1187S-187-10-5

2214S-141-19-1 ||

(10) 3412S-171-29-1

(11) 1544S-136-23-3 || 1382S-161-11-4 ||

(12)

49S-41-2-2

1625S-39-7-3 ||

(13)

(14)

(15)

800S--3

2061S-165-11-1

462S-66C-20-3 ||

558T-148-5-4

569S-4C-35-6 ||

2221S-195-3-2

1919S-144-11A-3 ||

PicoSL 3

16) $494 \mathrm{~S}-95-4-4$

1611T-41-49-1 |

1516S-55-13-3 ||

1952S-66-40-1 ||

234S-16-6-1

645S-156-10-2 ||

3141S-150-51-1 ||

1428S-129-19-1 |

1819S-176-4-1 ||

(17) $1469 S-150-34-3$ ||

945S-186-5-3 ||

1175S-164-5-1 ||

700S--2 ||

2196S-149-32-2 ||

1695S-128-24-2

3156S-194-27-4 ||

649S-98-11-1

838S- -2

(18)

114S-79-1-4 H.

\section{(95)-19-7-1}

1750S-161-18-3

93S-74-2-1

1614S-6-65-5 |

181S-60-6-3 H.

2075T-67-61-1 |

609S-149-6-3 ||

174S-103-6-3 ||

(19) 463S-66-21-1

1774S-167-25-2 ||

636S-144-2-3

(21) 2057S-172-23-3 ||

945S-186-5-5

1715S-152-35-3 ||

2217S-141-22-1 ||

mmu-mir-363

mmu-mir-92-2 |

mmu-mir-19b-2 |

mmu-mir-20b |

mmu-mir-106a |

MicroRNAs from miRBase

AY940616 «<

AY940614 «

AY940615 स

AY940617 |-

AY940618

AK084356

Mouse mRNAs from GenBank

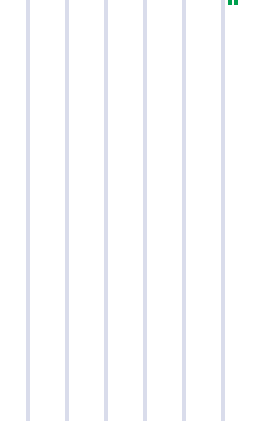

AK149675

$5 \mathrm{~kb}$

Figure I

Map of integration sites upstream of the mmu-mir- I 06a cistron. A map of the SL3-3 retroviral sites upstream of the mir- 106a cluster is displayed using the UCSC genome website browser (February 2006 version of the mm8 genome assembly). Insertion sites are depicted as vertical handlebars. Tumors assayed by quantitative PCR are numbered and noted in black text. 
A)

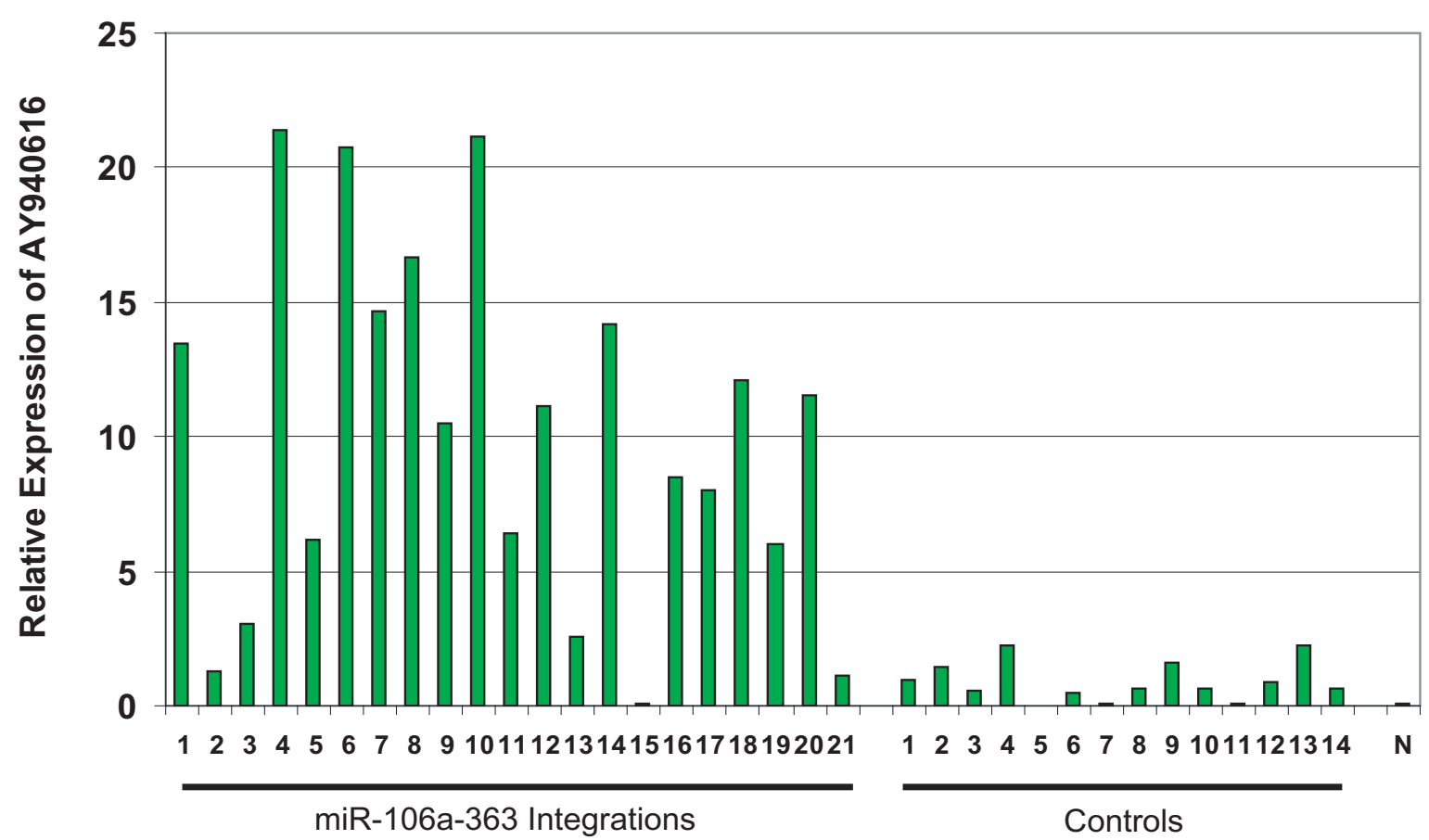

B)

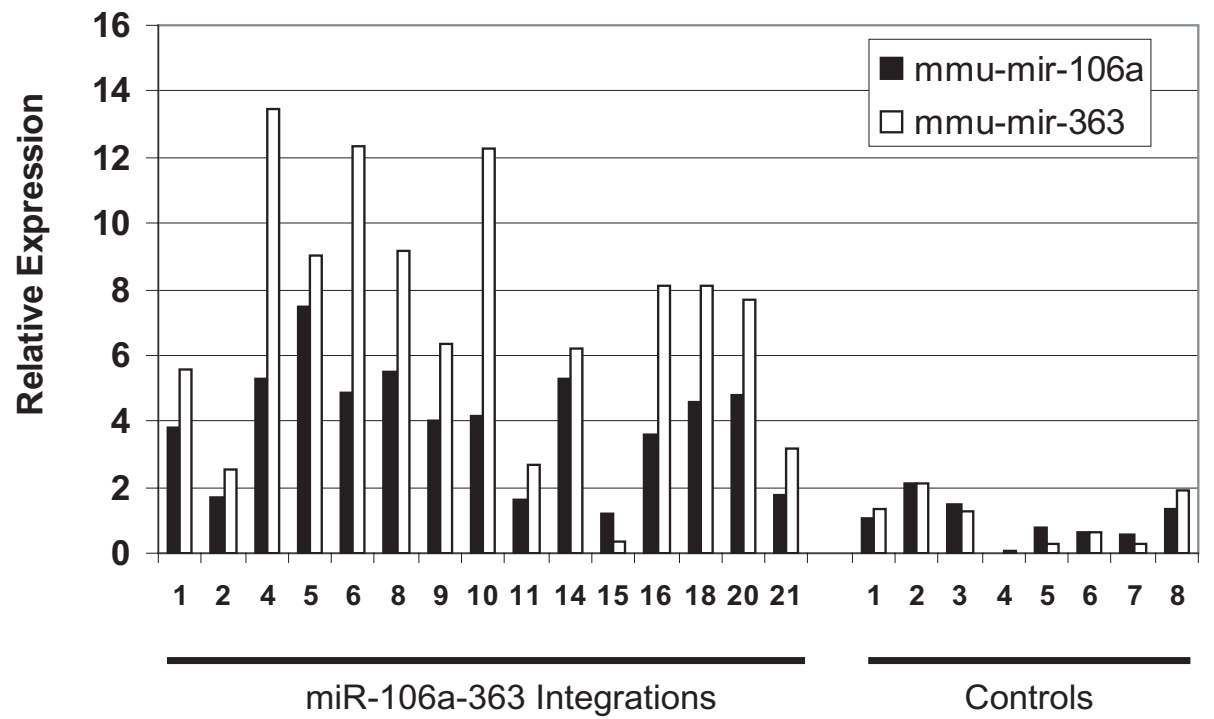

Figure 2

Expression of the primary transcript and mature species of the mmu-mir-106a cistron. Quantitative PCR data for tumors with integrations upstream of the mir-106a cistron. (A) Relative expression of AY940616 in tumors containing integration sites near the mir-106a miRNA cluster. Control tumors contain integration sites at locations in the genome other than the mir-106a region. "N" is cDNA generated from normal mouse spleen RNA (Ambion). (B) Relative expression of the mature species of mmu-mir-106a and mmu-mir-363 in tumors containing integration sites near the mir-106a cistron. Tumors are numbered as in Figure I. 
Table I: Assayed mmu-mir-106a cistron integrations

\begin{tabular}{|c|c|c|c|}
\hline \# & Tumor & Location & Orientation \\
\hline I & I759S-158-18 & chrX:48988832 & G-T- \\
\hline 2 & |890S-76-| & chrX:48988834 & G-T+ \\
\hline 3 & $718 S-68-23$ & chrX:48989486 & G-T+ \\
\hline 4 & I786S-166-32 & chrX:48989857 & G-T- \\
\hline 5 & $818 S-36-8$ & chrX:48989770 & G-T+ \\
\hline 6 & $553 S-122-16$ & chrX:48990068 & G-T- \\
\hline 7 & $1758 S-158-17$ & chrX:48989877 & G-T+ \\
\hline 8 & $1189 S-187-12$ & chrX:48989980 & G-T+ \\
\hline 9 & 3390T-I 28-37 & chrX:48990|80 & G-T- \\
\hline 10 & $34|2 S-| 7 \mid-29$ & chrX:48990। 92 & G-T+ \\
\hline 11 & I544S-136-23 & chrX:4899039| & G-T- \\
\hline 12 & $49 S-4 I-2$ & chrX:4899|005 & G-T- \\
\hline 13 & $800 \mathrm{~S}-$ & chrX:4899|024 & G-T+ \\
\hline 14 & 206IS-I65-II & chrX:4899| 259 & G-T- \\
\hline 15 & $462 S-66 C-20$ & chrX:4899|I59 & G-T+ \\
\hline 16 & $494 S-95-4$ & chrX:4899652। & G-T+ \\
\hline 17 & I469S-150-34 & chrX:48999846 & G-T+ \\
\hline 18 & 195S-19-7 & chrX:49000742 & G-T+ \\
\hline 19 & $463 S-66-21$ & chrX:49002308 & G-T+ \\
\hline 20 & $14 \mid 5 S$ & chrX:49004II 5 & G-T+ \\
\hline 21 & $2057 S-172-23$ & chrX:49005225 & G-T- \\
\hline
\end{tabular}

Retroviral insertion site locations (February 2006 version of the UCSC mm8 genome assembly) are noted by the basepair located just before the insertion. Orientation of the retrovirus is described using the following nomenclature. The gene transcript $(\mathrm{G})$ and retroviral tag $(T)$ is directly followed by a "+" for directionality of left to right or by a "-" for directionality of right to left on the chromosome. If the gene transcript is located to the left of the retroviral tag on the chromosome, the notation for the gene precedes the notation for the $\operatorname{tag}(G \pm T \pm)$. If the transcript is to the right of the retroviral tag on the chromosome the order is reversed $(T \pm G \pm)$. If the tag is located within the gene, by default the gene precedes the tag annotation.

in the Kis2 region cause overexpression not only of the primary RNA, but also of the mature species of the mir106 a cluster. This, in turn, suggests that the miRNA cluster can drive the development of $\mathrm{T}$ lymphomas. Although there is a possibility that these integrations also may affect the expression of other oncogenes and tumor suppressors in this region, our data clearly indicates a majority of these integrations induce the expression of the mir-106a cluster.

As the mir-106a cistron is a homolog of the oncogenic mir-17 cistron [16], it is not unexpected that mir-106a would also be involved tumorigenesis. Indeed, in human solid tumors, mir-106a expression is increased in colon, pancreas, and prostate tumors; and mir-92-2 expression is increased in pancreas, prostate, and stomach tumors [17]. Given the sequence similarity between the mir-17 and mir-106a cistrons, it is likely that these clusters have overlapping gene targets. In humans, the mir-106a cistron contains several paralogs to members of the mir-17 cistron including mir-17, mir-19b-1, and mir-92-1 [16], which are implicated in cancer development: overexpression of the mir-17 cluster accelerates lymphoma forma- tion from cells of mice overexpressing c-Myc [7]. The mir17 cluster is also overexpressed in human lung cancer [18]. However, in breast cancer cells, mir-17-5p expression is decreased; there it acts as a translational repressor of the oncogene AIB1 (amplified in breast cancer 1) [19], and in this context may formally act as a tumor suppressor.

It is well established that tumorigenesis is the result of accumulating several cooperating mutations that drive relentless proliferation and aid in metastases. Viral insertional mutagenesis, though perhaps not providing all the mutations necessary for a full-blown tumor, follows this multistep scenario. Although in general the superinfection barrier largely prevents multiple proviral integrations within the same cell, re-infection does happen over time. Because it is a rare event, such cells are selected over the others only when these integrations also give a growth advantage. As a consequence, in general, most viral insertions ("co-mutations") in a single tumor are thought to be causative in its formation. With the caveats of potential passenger genes and potential oligoclonality of tumors, co-mutation analysis may be a powerful way to find cooperating signaling pathways in tumorigenesis.

We detected multiple insertion sites in all of the tumor samples we assayed from the mir-106a cluster. Genes near common co-integration sites for these tumors include Ahi1, Evi5, and Gfi1, candidates previously appearing in retroviral screens [12], as well as PVT1, a noncoding RNA frequently amplified with myc [20]. A summary of all integration sites in the assayed tumors is listed in Table 2.

Through retroviral insertion in the mouse, we have discovered another potentially oncogenic microRNA cluster, mir-106a-363. Retroviral insertion caused significant overexpression of this microRNA cluster indicating its role in tumor development. This study further demonstrates the power of retrovirus insertion as a tool to discover new oncogenic noncoding RNAs.

\section{Competing interests}

The authors declare a financial interest in Picobella, LLC.

\section{Authors' contributions}

AML carried out the RNA isolation, quantitative PCR, expression data analysis, and drafted the manuscript. GB, LL, NC, and BBW carried out the tag recovery and identification. BBW and MW planned and directed the execution of the retroviral screen, the design of the study, and the writing of the manuscript.

\section{Acknowledgements}

This work was supported by NIH grant CA 100266 to MW, and Synergenics, LLC. We thank Dr. Clifford Wang for his technical advice and for his comments on the manuscript. 
Table 2: Summary of integrations in tumors assayed for the mmu-mir-106a cistron

\begin{tabular}{|c|c|c|c|c|c|}
\hline \multirow[b]{2}{*}{ Tumor \# } & \multirow[b]{2}{*}{ Tumor Name } & \multicolumn{4}{|c|}{ Gene located near insertion site } \\
\hline & & Location & Orientation & Abbr/Acc & Description \\
\hline 1 & $1759 S-158-18$ & chr18:78192824 & $\mathrm{G}+\mathrm{T}+$ & XM_973419 & \\
\hline 1 & |759S-|58-18 & chr7:73480330 & T-G+ & XM_978I27.I & \\
\hline 1 & I759S-I58-18 & chr5:107965444 & G-T- & Gfil & Growth factor independent I \\
\hline 2 & |890S-76-| & chr10:20756607 & $\mathrm{G}+\mathrm{T}-$ & Ahil & Jouberin \\
\hline 2 & |890S-76-| & chr7:| 44921997 & T-G- & Tpcn2 & Two pore segment channel 2 \\
\hline 2 & |890S-76-। & chr15:61868694 & $\mathrm{G}+\mathrm{T}+$ & PVTI & Plasmacytoma variant translocation I \\
\hline 3 & $7185-68-23$ & chr17:29126185 & $\mathrm{G}+\mathrm{T}-$ & Fgd2 & FYVE, RhoGEF and PH domain containing 2 \\
\hline 3 & $7185-68-23$ & chrl I:5819233 & G-T- & Gck & Glucokinase \\
\hline 3 & $7185-68-23$ & chrl I:66037536 & G-T- & Gm879 & Gene model 879 \\
\hline 4 & $1786 S-166-32$ & chr5:I1657/294 & G-T- & Ccdc60 & Coiled-coil domain containing 60 \\
\hline 4 & |786S-166-32 & chr5:|0797738| & $\mathrm{T}+\mathrm{G}-$ & Evi5 & Ecotropic viral integration site 5 \\
\hline 5 & $818 S-36-8$ & $\operatorname{chr|8:534877|}$ & G-T- & Zfp438 & Zinc finger protein 438 \\
\hline 5 & $818 S-36-8$ & chr5:15380286 & T-G+ & Cacna2dl & $\begin{array}{l}\text { Calcium channel, voltage-dependent, alpha2/delta } \\
\text { subunit I }\end{array}$ \\
\hline 6 & $553 S-122-16$ & chr5:107957997 & G-T- & Gfil & Growth factor independent I \\
\hline 6 & $553 S-122-16$ & $\operatorname{chrl5:62006727}$ & $\mathrm{G}+\mathrm{T}-$ & PVTI & Plasmacytoma variant translocation I \\
\hline 7 & |758S-|58-| 7 & chr5:II542/225 & G-T- & 2410014 A08Rik & Hypothetical protein LOCI09154 \\
\hline 7 & $1758 S-\mid 58-17$ & chr5:107968359 & G-T+ & Gfil & Growth factor independent I \\
\hline 7 & |758S-|58-17 & chrl7:46990647 & G-T- & Tbn & Taube nuss \\
\hline 8 & $1189 S-187-12$ & chr5:107970686 & G-T+ & Gfil & Growth factor independent I \\
\hline 8 & $1189 S-187-12$ & chr9:20880975 & G-T- & Tyk2 & Tyrosine kinase 2 \\
\hline 8 & $1189 S-187-12$ & chrl 5:63293889 & $\mathrm{T}+\mathrm{G}-$ & XM_I39402 & \\
\hline 8 & $11895-187-12$ & chrl 9:55328465 & $\mathrm{G}+\mathrm{T}-$ & Acsl5 & Acyl-CoA synthetase long-chain family member 5 \\
\hline 9 & $3390 \mathrm{~T}-128-37$ & chr2:117|24415 & G-T+ & Rasgrpl & RAS guanyl releasing protein I \\
\hline 9 & $3390 \mathrm{~T}-128-37$ & chr7:113933436 & G-T+ & Rras2 & Related RAS viral ( $r$-ras) oncogene homolog 2 \\
\hline 9 & $3390 \mathrm{~T}-128-37$ & chr7:II36364I2 & $\mathrm{G}+\mathrm{T}-$ & Spon I & Spondin I, (f-spondin) extracellular matrix protein \\
\hline 9 & $3390 \mathrm{~T}-128-37$ & chr10:20761837 & $\mathrm{G}+\mathrm{T}-$ & Ahil & Jouberin \\
\hline 10 & $34|2 S-| 7 \mid-29$ & $\operatorname{chr} 10: 20781072$ & $\mathrm{G}+\mathrm{T}-$ & Ahil & Jouberin \\
\hline 11 & I544S-136-23 & chrl3_random:67900 & $\mathrm{G}+\mathrm{T}+$ & NM_I75538.2 & RIKEN cDNA EI30304F04 gene \\
\hline 11 & I544S-136-23 & chr9:36980695 & G-T+ & Slc37a2 & $\begin{array}{l}\text { Solute carrier family } 37 \text { (glycerol-3-phosphate } \\
\text { transporter), member } 2\end{array}$ \\
\hline 11 & I544S-136-23 & chr10:58965।85 & G-T+ & NM_001033259.1 & RIKEN cDNA DI30073L02 gene \\
\hline 12 & $49 S-4 I-2$ & chr2:72016508 & $\mathrm{G}+\mathrm{T}+$ & Rapgef4 & Rap guanine nucleotide exchange factor (GEF) 4 \\
\hline 12 & $49 S-41-2$ & chr17:47006160 & G-T- & Tbn & Taube nuss \\
\hline 13 & $800 \mathrm{~S}-$ & chr7:58627272 & $\mathrm{G}+\mathrm{T}-$ & Atpl0a & ATPase, class $V$, type I0A \\
\hline 13 & $800 \mathrm{~S}-$ & $\operatorname{chr} 16: 94677486$ & G-T- & Dscr3 & Down syndrome critical region gene 3 \\
\hline 14 & $206 \mid S-165-11$ & chrl4:771727/2 & $T+G-$ & Akapll & A kinase (PRKA) anchor protein II \\
\hline 14 & $206|S-| 65-||$ & chr5: 107977456 & $\mathrm{~T}+\mathrm{G}-$ & Evi5 & Ecotropic viral integration site 5 \\
\hline 14 & $206|S-| 65-I \mid$ & chrl 7:29|25847 & $\mathrm{G}+\mathrm{T}-$ & Fgd2 & FYVE, RhoGEF and PH domain containing 2 \\
\hline 15 & $462 S-66 C-20$ & chr2:26317278 & G-T+ & Notchl & Notch gene homolog I (Drosophila) \\
\hline 15 & $462 S-66 C-20$ & chr10:20779700 & $\mathrm{G}+\mathrm{T}-$ & Ahil & Jouberin \\
\hline 15 & $462 S-66 C-20$ & $\operatorname{chr|2:8643529|}$ & $\mathrm{G}+\mathrm{T}-$ & XM_988509 & \\
\hline 16 & $494 S-95-4$ & chr I0:120923128 & $\mathrm{T}+\mathrm{G}-$ & Tbkl & TANK-binding kinase I \\
\hline 16 & $494 S-95-4$ & chr|4:121149083 & $\mathrm{G}+\mathrm{T}+$ & Phgdhll & Phosphoglycerate dehydrogenase like I \\
\hline 16 & $494 S-95-4$ & chrl:I39964759 & G-T+ & Ptprc & Protein tyrosine phosphatase, receptor type, C \\
\hline 17 & |469S-|50-34 & $\operatorname{chr} 16: 48708118$ & $\mathrm{G}+\mathrm{T}+$ & LOC432823 & \\
\hline 17 & I469S-150-34 & chr5: 107979863 & $T+G-$ & Evi5 & Ecotropic viral integration site 5 \\
\hline 17 & 1469S-150-34 & $\operatorname{chr} 12: 86550688$ & $\mathrm{G}+\mathrm{T}+$ & Jundm2 & Jun dimerization protein 2 \\
\hline 18 & 195S-19-7 & $\operatorname{chr} 10: 20744863$ & $\mathrm{G}+\mathrm{T}-$ & Ahil & Jouberin \\
\hline 19 & $463 S-66-21$ & chr4:98038839 & $\mathrm{G}+\mathrm{T}-$ & Inadl & InaD-like (Drosophila) \\
\hline 19 & $463 S-66-21$ & chr5:107968538 & G-T+ & Gfil & Growth factor independent I \\
\hline 19 & $463 S-66-21$ & chrl 7:46998042 & G-T- & Tbn & Taube nuss \\
\hline 19 & $463 S-66-21$ & chrl:I37623944 & $\mathrm{G}+\mathrm{T}-$ & Tnnil & Troponin I, skeletal, slow I \\
\hline 20 & $1415 S-$ & chrl5:61998664 & $\mathrm{G}+\mathrm{T}+$ & PVTI & Plasmacytoma variant translocation I \\
\hline 20 & $14 \mid 5 S$ & chrl4:68239584 & G-T+ & Slc $25 \mathrm{a} 37$ & Solute carrier family 25, member 37 \\
\hline 20 & $1415 S-$ & chr|4:7827|47| & $\mathrm{G}+\mathrm{T}-$ & ElfI & E74-like factor I \\
\hline 21 & $2057 S-172-23$ & chr|5:798328II & G-T+ & Pdgfb & platelet derived growth factor, B polypeptide \\
\hline 21 & $2057 S-172-23$ & chrl 3:52820992 & T-G- & Auh & AU RNA binding protein/enoyl-coenzyme A hydratase \\
\hline
\end{tabular}

Additional retroviral insertion sites recovered from tumors containing an insertion site upstream of the mir-106a cistron. Retroviral insertion site locations and orientations are notated as in Table I. Nearby genes to the insertion sites are also listed. 


\section{References}

I. Uren AG, Kool J, Berns A, van Lohuizen M: Retroviral insertional mutagenesis: past, present and future. Oncogene 2005, 24:7656-7672.

2. Suzuki T, Shen H, Akagi K, Morse HC, Malley JD, Naiman DQ, Jenkins NA, Copeland NG: New genes involved in cancer identified by retroviral tagging. Nat Genet 2002, 32:I66-I74.

3. Mikkers H, Allen J, Knipscheer P, Romeijn L, Hart A, Vink E, Berns A: High-throughput retroviral tagging to identify components of specific signaling pathways in cancer. Nat Genet 2002, 32:153-159.

4. Li J, Shen H, Himmel KL, Dupuy AJ, Largaespada DA, Nakamura T, Shaughnessy JD Jr., Jenkins NA, Copeland NG: Leukaemia disease genes: large-scale cloning and pathway predictions. Nat Genet 1999, 23:348-353.

5. Wang CL, Wang BB, Bartha G, Li L, Channa N, Klinger M, Killeen N, Wabl M: Activation of an oncogenic microRNA cistron by provirus integration. Proc Natl Acad Sci U S A 2006, |03:|8680-|8684.

6. Shamovsky I, Ivannikov M, Kandel ES, Gershon D, Nudler E: RNAmediated response to heat shock in mammalian cells. Nature 2006, 440:556-560.

7. He L, Thomson JM, Hemann MT, Hernando-Monge E, Mu D, Goodson S, Powers S, Cordon-Cardo C, Lowe SW, Hannon G], Hammond SM: A microRNA polycistron as a potential human oncogene. Nature 2005, 435:828-833.

8. Calin GA, Sevignani C, Dumitru CD, Hyslop T, Noch E, Yendamuri S, Shimizu M, Rattan S, Bullrich F, Negrini M, Croce CM: Human microRNA genes are frequently located at fragile sites and genomic regions involved in cancers. Proc Natl Acad Sci U S A 2004, I 01 :2999-3004.

9. O'Donnell KA, Wentzel EA, Zeller KI, Dang CV, Mendell JT: c-Mycregulated microRNAs modulate E2FI expression. Nature 2005, 435:839-843.

10. Costinean S, Zanesi N, Pekarsky Y, Tili E, Volinia S, Heerema N, Croce CM: Pre-B cell proliferation and lymphoblastic leukemia/high-grade lymphoma in $E(\mathrm{mu})-\mathrm{miR} I 55$ transgenic mice. Proc Natl Acad Sci U S A 2006, 103:7024-7029.

II. Lenz J, Crowther R, Klimenko S, Haseltine W: Molecular cloning of a highly leukemogenic, ecotropic retrovirus from an AKR mouse. J Virol 1982, 43:943-95I.

12. Akagi K, Suzuki T, Stephens RM, Jenkins NA, Copeland NG: RTCGD: retroviral tagged cancer gene database. Nucleic Acids Res 2004, 32:D523-7.

13. Hwang HC, Martins CP, Bronkhorst Y, Randel E, Berns A, Fero M, Clurman BE: Identification of oncogenes collaborating with p27Kip I loss by insertional mutagenesis and high-throughput insertion site analysis. Proc Natl Acad Sci U S A 2002, 99: I I293-II298.

14. Landais S, Quantin R, Rassart E: Radiation leukemia virus common integration at the Kis2 locus: simultaneous overexpression of a novel noncoding RNA and of the proximal Phf6 gene. J Virol 2005, 79: I |443- I |456.

15. Chen C, Ridzon DA, Broomer AJ, Zhou Z, Lee DH, Nguyen JT, Barbisin M, Xu NL, Mahuvakar VR, Andersen MR, Lao KQ, Livak KJ, Guegler KJ: Real-time quantification of microRNAs by stem-loop RT-PCR. Nucleic Acids Res 2005, 33:el 79.

16. Tanzer A, Stadler PF: Molecular evolution of a microRNA cluster. J Mol Biol 2004, 339:327-335.

17. Volinia S, Calin GA, Liu CG, Ambs S, Cimmino A, Petrocca F, Visone R, lorio M, Roldo C, Ferracin M, Prueitt RL, Yanaihara N, Lanza G, Scarpa A, Vecchione A, Negrini M, Harris CC, Croce CM: A microRNA expression signature of human solid tumors defines cancer gene targets. Proc Natl Acad Sci U S A 2006, I03:2257-226I.

18. Hayashita $Y$, Osada H, Tatematsu Y, Yamada H, Yanagisawa K, Tomida S, Yatabe Y, Kawahara K, Sekido Y, Takahashi T: A polycistronic microRNA cluster, miR-17-92, is overexpressed in human lung cancers and enhances cell proliferation. Cancer Res 2005, 65:9628-9632

19. Hossain A, Kuo MT, Saunders GF: Mir-I7-5p Regulates Breast Cancer Cell Proliferation by Inhibiting Translation of AIB I mRNA. Mol Cell Biol 2006, 26:8191-8201.

20. Shtivelman E, Bishop JM: The PVT gene frequently amplifies with MYC in tumor cells. Mol Cell Biol I 989, 9: I | 48-I I 54.

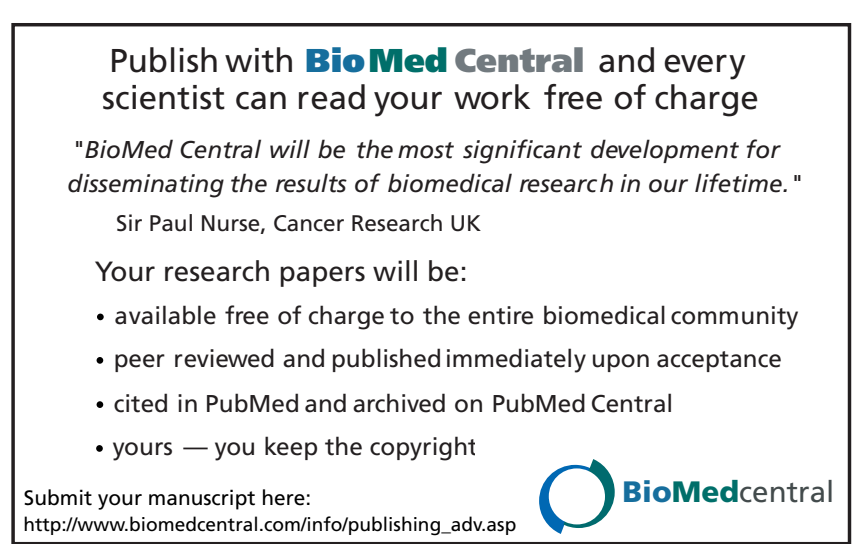

